\title{
Modulation of pain threshold by virtual body ownership
}

\author{
M. Martini ${ }^{1,2}$, D. Perez-Marcos ${ }^{1,2}$, M.V. Sanchez-Vives ${ }^{1,2,3,4}$ \\ 1 Institut d'Investigacions Biomèdiques August Pi i Sunyer (IDIBAPS), Barcelona, Spain \\ 2 EVENT-Lab, Facultat de Psicologia, Universitat de Barcelona, Spain \\ 3 Institució Catalana Recerca i Estudis Avançats (ICREA), Barcelona, Spain \\ 4 Departament de Psicologia Bàsica, Universitat de Barcelona, Spain
}

Correspondence

Matteo Martini

E-mail: mmartini@clinic.ub.es

\section{Funding sources}

This research was supported by FP7 FET EU collaborative project BEAMING (248620).

Conflicts of interest

None declared.

Accepted for publication

13 December 2013

doi:10.1002/j.1532-2149.2014.00451.x

\begin{abstract}
Background: Appropriate sensorimotor correlations can result in the illusion of ownership of exogenous body parts. Nevertheless, whether and how the illusion of owning a new body part affects human perception, and in particular pain detection, is still poorly investigated. Recent findings have shown that seeing one's own body is analgesic, but it is not known whether this effect is transferable to newly embodied, but exogenous, body parts. In recent years, results from our laboratory have demonstrated that a virtual body can be felt as one's own, provided realistic multisensory correlations.
\end{abstract}

Methods: The current work aimed at investigating the impact of virtual body ownership on pain threshold. An immersive virtual environment allowed a first-person perspective of a virtual body that replaced the own. Passive movement of the index finger congruent with the movement of the virtual index finger was used in the 'synchronous' condition to induce ownership of the virtual arm. The pain threshold was tested by thermal stimulation under four conditions: (1) synchronous movements of the real and virtual fingers; (2) asynchronous movements; (3) seeing a virtual object instead of an arm; and (4) not seeing any limb in real world.

Results: Our results show that, independently of attentional and stimulus adaptation processes, the ownership of a virtual arm per se can significantly increase the thermal pain threshold.

Conclusions: This finding may be relevant for the development and improvement of digital solutions for rehabilitation and pain treatment.

\section{Introduction}

Virtual reality (VR) technology represents a versatile tool for different areas of research since it allows the creation of sensory environments that can be replicated identically across experiments and that are under the full control of the experimenter (SanchezVives and Slater, 2005). In particular, VR offers a novel and valuable resource for psychological and medical rehabilitation purposes. In pain management, immersive VR has been reported by Hoffman and colleagues as an adjunctive analgesic treatment of pain for burninjured adolescent (Hoffman et al., 2000a) and adult patients (Hoffman et al., 2000b). Other studies refer to the employment of VR as a method for tackling phantom limb pain (Murray et al., 2007; Cole et al., 2009) and for the diagnosis and treatment of complex regional pain syndromes (Sato et al., 2010; Llobera et al., 2013a). The analgesic effect derived from the utilization of VR often stems from its power to draw attentional resources away from the hurting body part (Malloy and Milling, 2010). It has been shown that VR is highly effective since it can provide an alternative reality, fully immersive and interactive. Indeed, distraction per se has been recognized as a powerful factor in lowering pain ratings outside VR (Bantick et al., 2002). Nonetheless, not all the analgesic effects due to psychological factors rely upon sheer attentional 


\section{What's already known about this topic?}

- Realistic sensorimotor correlations induce the illusion of ownership of virtual body parts.

- Recent findings have demonstrated that seeing one's own body is analgesic, but it is not known whether this effect is transferable to newly embodied body parts.

\section{What does this study add?}

- The current study demonstrates that heat pain threshold significantly increases following ownership of a digital body in virtual reality. Virtual reality itself or just looking at a non-embodied digital body does not yield the same effect.

modulation. Studies conducted in healthy subjects have shown a modulatory effect of the vision of the body on experimentally induced pain. For instance, Longo and colleagues reported a decrease in the pain ratings when the participants looked at their own body but not when they looked either at a noncorporeal object or at someone else's body, suggesting that the vision of one's own body may have a local analgesic effect (Longo et al., 2009, 2012). Another study has shown that the manipulation of one's hand or limb size affects pain, such that seeing the hand smaller decreases pain, while seeing the hand larger increases it (Mancini et al., 2011).

Despite the substantial evidence showing that a fake or a virtual body part can be incorporated into one's body image (i.e., embodied) (Botvinick and Cohen, 1998; Slater et al., 2008; Sanchez-Vives et al., 2010), and that looking at one's body can modulate pain perception (Longo et al., 2009), to our knowledge, only two studies have investigated so far the relationship between pain and embodiment, with contradictory results (Hansel et al., 2011; Mohan et al., 2012). In one of these studies (Hansel et al., 2011), the authors assessed pressure pain threshold on the index finger while using an out-of-body experience paradigm (third-person perspective approach). They reported that just the vision of the mannequin standing in front of the participants led to higher pain thresholds, independently of the identification with it (Hansel et al., 2011). In the other study, no effect of the rubber hand illusion was found on pain perception (Mohan et al., 2012).

Hence, it remains unclear whether the effect of seeing one's own body on pain perception holds true when embodying new dummy or virtual bodies. In the current study, we investigate the effects of virtual body ownership on the heat pain threshold. To create the illusion of ownership of the virtual body, we use multisensory correlations and first-person perspective with respect to a virtual body.

\section{Methods}

\subsection{Participants}

Thirty-two right-handed healthy participants (19 females, mean \pm SD age: $23.9 \pm 5.7$ ) were recruited for the experiment. They had normal or corrected-to-normal vision and no history of neurological or psychological disorders. Also, any condition potentially interfering with pain sensitivity (e.g., drug intake) was considered as a further exclusion criterion. Upon arrival at the laboratory, they were asked to read and sign a consent form, and the experiment was carried out in accordance with the regulations of our ethics committee (Comité Ético de Investigación Clínica de la Corporación Sanitaria Hospital Clínic de Barcelona). All participants received a monetary reimbursement for their participation ( $10 €)$.

Since the purpose of the study was to examine the effects of virtual body ownership on pain threshold, a prerequisite was that participants were able to experience that body illusion (Ehrsson et al., 2005). Therefore, only those participants that experienced the illusion, i.e., those scoring between 5 and 7 in the question referring to virtual body ownership (see the Questionnaire section), were considered for this study. Thus, data from 24 healthy right-handed participants ( 14 females, mean \pm SD age: $25.5 \pm 5.8$ ) were finally taken into consideration. This represents $75 \%$ of the initial sample, which is comparable to the percentage obtained previously in experiments with rubber (Ehrsson et al., 2005) and virtual hand illusions (Perez-Marcos et al., 2012).

\subsection{Virtual reality system}

The stereoscopic head-mounted display (HMD) was an NVIS SX111 with a resolution of $1280 \times 1024$ pixels per eye and a total field of view of $111^{\circ} \times 64^{\circ}$, displayed at $60 \mathrm{~Hz}$. The head tracking was realized with a 6-DOF Intersense IS-900 device (InterSense, Billerica, MA, USA). Finger tracking was permitted by attaching two markers to the participant's finger. These markers were constantly tracked by 12 infrared Optitrack cameras and their coordinates in the space were computed with the Arena software (NaturalPoint, Corvallis, OR, USA). Hence, when the participant's finger was moved, the avatar's finger could move accordingly, mimicking exactly the same movements at the same time. The virtual environment was programmed using the XVR system (Tecchia et al., 2010) and the virtual body using the HALCA library (Gillies and Spanlang, 2010). Figures 1 and 2 show how the real and the virtual environments looked like.

Noise isolation was ensured by the administration of pink noise through a surround audio system (Creative Technology 


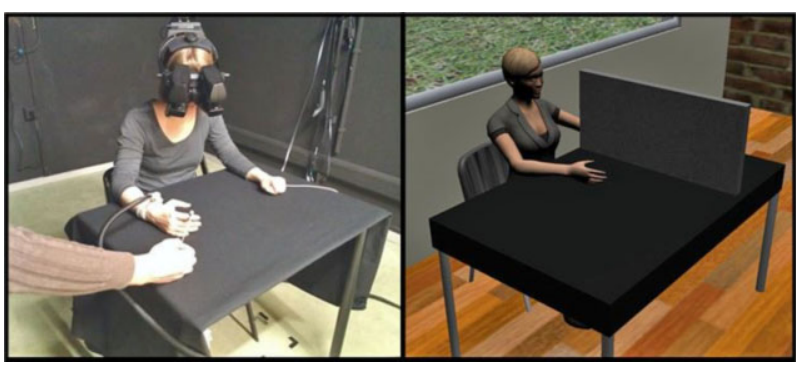

Figure 1 Illustrative picture of the real environment (left panel) and of the virtual environment with the female avatar (right panel).

Ltd, Creative Resource, Singapore), with a constant volume set at $65 \mathrm{~dB}$. Figure $2 \mathrm{~A}$ and $\mathrm{B}$ shows the virtual environments for the different VR conditions from a top view.

\subsection{Thermal stimulation and temperature}

Thermal heat stimuli were delivered by means of a SomedicThermotest machine (Somedic, Stockholm, Sweden) with a $2.5 \mathrm{~cm} \times 5.0 \mathrm{~cm}$ thermode tied with a Velcro strap on the forearm, close to the radius bone (see Fig. 2D). Pain thresholds were assessed with the method of limits (Yarnitsky et al., 1995). The probe temperature was increased from normal skin temperature (constant baseline temperature $=31{ }^{\circ} \mathrm{C}$ ) at $2{ }^{\circ} \mathrm{C} / \mathrm{s}$. Participants pressed a button with their left hand as soon as they perceived the stimulation as being painful. Immediately after pushing the kill-switch button, the probe temperature rapidly decreased to the baseline temperature. For safety reasons, maximal temperature was set at $50{ }^{\circ} \mathrm{C}$.

Skin temperatures on both the forearm (next to the thermode) and the hand (on the first dorsal interosseous) were measured with two Type K (TF-500) thermocouple probes linked to a PCE-T 390 digital thermometer (PCE Instruments, Meschede, Germany), with a resolution of $0.1{ }^{\circ} \mathrm{C}$ and a sampling rate of $0.5 \mathrm{~Hz}$. All temperatures were continuously monitored. The analogue data from the sensors of the thermometer were acquired with an NI-6008 card (National Instruments Corporation, Austin, TX, USA) and the values were saved in MatLab Simulink (The MathWorks Inc., Natick, MA, USA).

\subsection{Procedure}

Participants sat comfortably on a chair with both arms resting on a table covered with a black cloth, as shown in Figs. 1 and 2. The arms were in a straight but rested posture and were $60 \mathrm{~cm}$ apart. Before entering the VR, participants were given three heat stimuli to familiarize them with the heat ramps.

As the subject donned the HMD, the rooms' lights were turned off and the pink noise played. The HMD allowed participants to experience an immersive virtual environment around them and to see a virtual body collocated with their own from a first-person perspective (Fig. 2A). When they looked down towards their own body, they could see the virtual body in place of their real own body. Before the start of each VR condition, participants were given approximately 1 min to familiarize with the virtual room and with the virtual body. The experiment consisted of four different conditions:

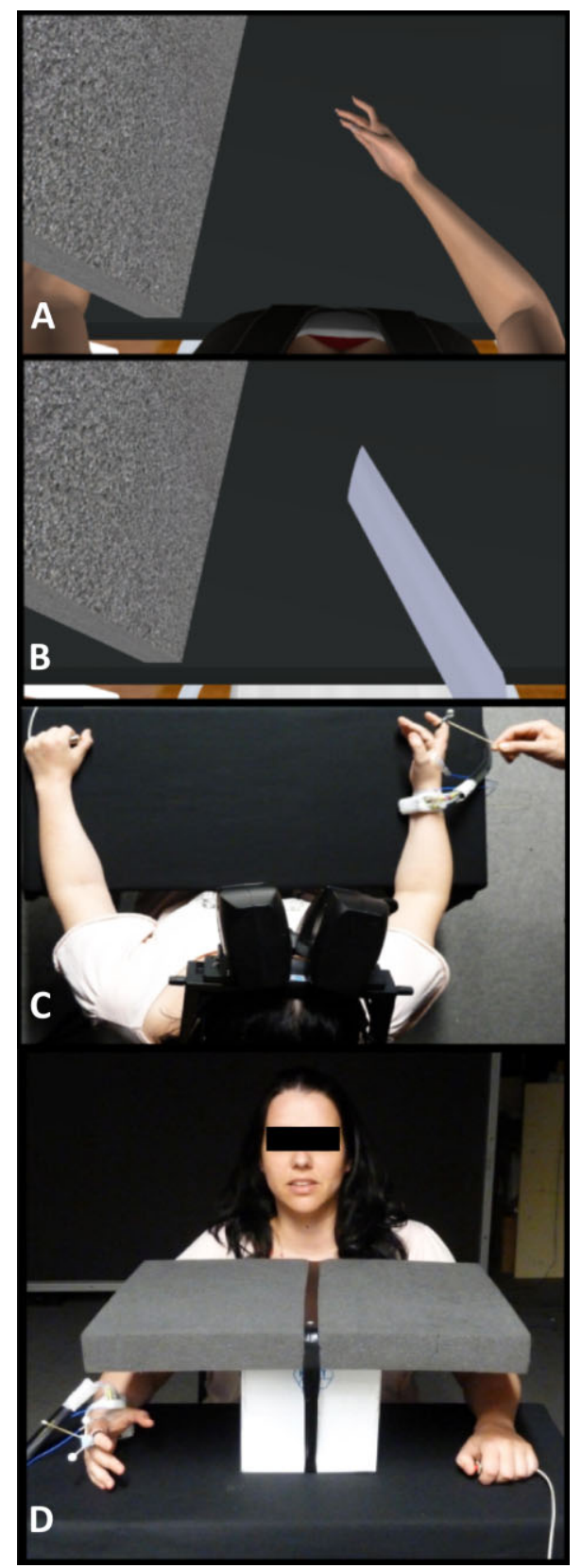

Figure 2 Experimental set-up: the avatar's body used in the asynchronous and synchronous conditions (A), and the non-corporeal object in the 'control inside' condition (B) are visualized through the head-mounted display, while one experimenter moves the participant's finger (C). In the 'control outside' condition, the sight of the limbs was prevented by using a foam cover (D). 
(1) A control condition run outside VR ('control outside', or ' $\mathrm{CO}^{\prime}$ ) served as a baseline. Participants were asked to look at a fixation mark placed on top of a foam cover, which prevented them from seeing their limbs (Fig. 2D).

(2) A control condition run within VR ('control inside' or ' $\mathrm{CI}^{\prime}$ ), where no virtual body was present. Instead, a noncorporeal object (an oblique cylinder) appeared on the table (Fig. 2B). Participants were asked to look at the tip of the cylinder, which corresponded to the place where the avatar's fingers were in the virtual-body condition.

(3) A condition within VR, where the avatar's index finger moved independently from the real finger ('asynchronous', or 'A' condition).

(4) A condition within VR, where the avatar's index finger moved in accordance with the real finger ('synchronous', or ' $\mathrm{S}$ ' condition).

Both in the A and in the S conditions, participants were asked to focus upon the finger movements. The virtual right arm appeared bent at about 41 degrees away from the real arm and towards the body midline (Fig. 2A). This procedure was meant to hamper the illusion of ownership in the $\mathrm{A}$ condition, which may have occurred by simply matching the collocation of one's limb with the avatar's limb (Slater et al., 2010). Importantly, both the virtual hand and the forearm were always kept in the field of view of the participants. In all conditions, an experimenter constantly moved the participant's right index finger in a flexion-extension fashion. This passive movement was meant to provide the proprioceptive feedback without calling into play the role of agency induced by active movement (Kalckert and Ehrsson, 2012). Characteristic of the S condition was that correspondence between the visual, motor and the proprioceptive inputs provided the experience of 'embodying' the avatar's limb, a phenomenon already documented as effective for inducing the virtual hand illusion (Sanchez-Vives et al., 2010). All participants completed the four conditions, with the order of the conditions balanced across participants. Each condition started with the participant looking at the indicated point for $20 \mathrm{~s}$. Then, the finger movement started, the first heat stimulus provided $20 \mathrm{~s}$ later. The inter-stimulus interval was set at $60 \mathrm{~s}$ and three heat ramps were provided for each condition.

\subsection{Questionnaire}

After each condition, the HMD was removed and the participants were given a questionnaire in Spanish (Table 1), which included different questions to evaluate anxiety, attention, presence in the virtual environment and body ownership for all given conditions. Each item was measured along a 7-point Likert scale. The order of the items in the questionnaire was randomized among subjects.

\subsection{Data handling}

Skin temperatures and pain thresholds were all recorded in ${ }^{\circ} \mathrm{C}$. For each condition, hand/arm skin temperatures and pain thresholds were recorded. Despite the proximity of the
Table 1 The questionnaire administered to the subjects, sorted by category.

\begin{tabular}{|c|c|}
\hline Category & During the current condition. .. \\
\hline Ownership & $\begin{array}{l}\text { 1. I felt as if the virtual right arm was my own right arm } \\
\text { (total disagreement }=1 \text {; total agreement }=7 \text { ). }{ }^{\text {a }} \\
\text { 2. I felt as if the virtual right hand was my own right } \\
\text { hand (total disagreement }=1 \text {; total agreement }=7 \text { ). }\end{array}$ \\
\hline Presence & $\begin{array}{l}\text { 3. I had a strong feeling of being in the lab (1); in the } \\
\text { virtual room (7). }{ }^{\text {a }}\end{array}$ \\
\hline Anxiety & 4. I felt totally relaxed (1); totally anxious (7)., a,b \\
\hline Attention & $\begin{array}{l}\text { 5. My attention was totally focussed on other things (for } \\
\text { example on what I was watching) (1); totally on the } \\
\text { thermal stimulus (7).., }\end{array}$ \\
\hline
\end{tabular}

Items 1-5 were used for both $\mathrm{A}$ and $\mathrm{S}$ conditions.

aUsed in the $\mathrm{Cl}$ condition. The term 'virtual right arm' was replaced by the term 'cylinder' (item 1).

bused in the CO condition.

arm sensor to the thermode, the recorded arm skin temperature was not influenced by the increasing heat ramps. The temperature at which participants pressed the button to stop the thermal stimulation was considered as the pain threshold (see above).

All variables were normally distributed according to both the Kolmogorov-Smirnov and the Lilliefors tests (all $\left.p^{\prime} \mathrm{s}>0.05\right)$. One-way repeated-measures analysis of variance (ANOVA) (factor: 'Condition' with 4 levels) was conducted on mean pain thresholds, as well as on the arm and hand skin temperature separately. Post-hoc analysis after one-way ANOVAs was conducted with Newman-Keuls tests.

Being obtained from an ordinal scale, the scores reported for the same variable (item) of the questionnaire in each condition were subjected to non-parametric Friedman ANOVAs. Post-hoc analysis with Wilcoxon matched-pairs tests was conducted with a Bonferroni correction applied for the number of possible comparisons. This resulted in a significance level set at $p<0.008$ for anxiety and attention scores, and at $p<0.016$ for cylinder/arm, cylinder/hand ownership and the level of presence.

Statistical comparisons between conditions were conducted with STATISTICA (StatSoft, Inc., Tulsa, OK, USA).

\section{Results}

\subsection{Pain thresholds and skin temperature}

The heat pain thresholds and skin temperature were measured for the four experimental conditions (CO, CI, A, S). Group mean pain thresholds for each condition and skin temperatures are reported in Table 2. The one-way ANOVA on the mean pain thresholds revealed a significant effect of the factor 'Condition' $\left(F_{3,69}=4.36 ; p=0.007\right)$, indicating that participant's pain threshold was differently affected under our experimental conditions. The Newman-Keuls post- 
Table 2 Pain thresholds and skin temperatures (mean \pm standard error) reported per condition.

\begin{tabular}{llll}
\hline Condition & $\begin{array}{l}\text { Pain } \\
\text { threshold }\left({ }^{\circ} \mathrm{C}\right)\end{array}$ & $\begin{array}{l}\text { Arm temperature } \\
\left({ }^{\circ} \mathrm{C}\right)\end{array}$ & $\begin{array}{l}\text { Hand temperature } \\
\left({ }^{\circ} \mathrm{C}\right)\end{array}$ \\
\hline $\mathrm{CO}$ & $43.08 \pm 0.40$ & $30.72 \pm 0.41$ & $30.38 \pm 0.51$ \\
$\mathrm{Cl}$ & $43.29 \pm 0.44$ & $30.92 \pm 0.39$ & $30.55 \pm 0.54$ \\
$\mathrm{~A}$ & $43.50 \pm 0.38$ & $30.77 \pm 0.36$ & $30.35 \pm 0.50$ \\
$\mathrm{~S}$ & $43.74 \pm 0.40$ & $30.77 \pm 0.38$ & $30.32 \pm 0.52$ \\
\hline
\end{tabular}

hoc test revealed that the only condition reporting significant difference with the others was the S condition, namely when the ownership of the avatar's arm occurred. Indeed, only in this condition the mean threshold was significantly higher with respect to either the CO condition $(p=0.006)$ or the CI condition ( $p=0.038$; see Fig. 3 ). No other comparison was found to be significant (S vs. A: $p=0.22$; A vs. CO: $p=0.092$; A vs. CI: $p=0.21$; CI vs. CO: $p=0.39)$. These results suggest that only when participants had the illusion to own the virtual body their pain threshold was effectively higher, while just the vision of an avatar's body or of an object replacing the body did not yield any statistically relevant difference.

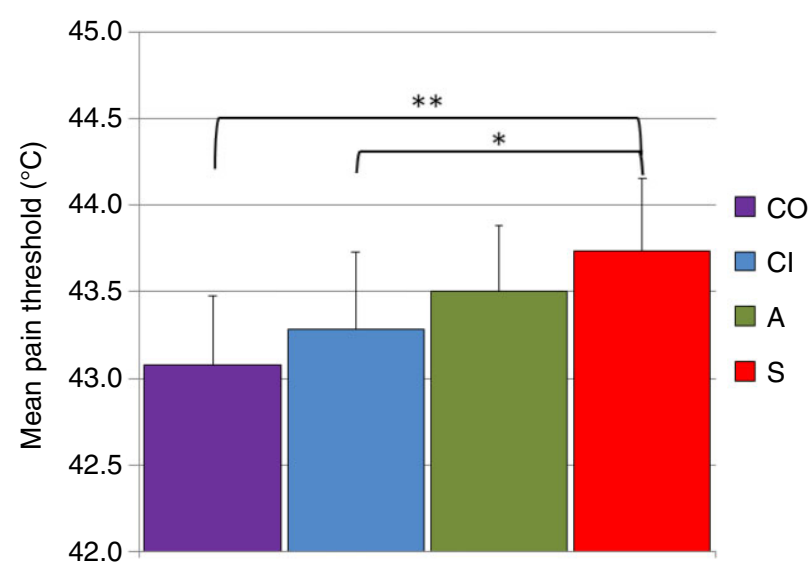

Figure 3 Mean and standard error of the pain thresholds reported by the participants in each condition. Purple, light blue, green and red are associated with the 'control outside', 'control inside', 'asynchronous' and 'synchronous' conditions ( $\mathrm{CO}, \mathrm{Cl}, \mathrm{A}$ and $\mathrm{S}$, respectively). Asterisks indicate significant comparisons ( ${ }^{\star} p<0.05 ;{ }^{*} p<0.001$ ).
With respect to skin temperature, the one-way repeated-measures ANOVA showed no significant effect of the factors for the $\operatorname{arm}\left(F_{3,69}=0.73 ; p=0.53\right)$ or for the hand ('Condition': $F_{3,60}=0.63 ; p=0.60$ ), revealing a lack of modulatory effect on skin temperatures by the ownership of the virtual arm and hand under the present experimental design.

\subsection{Questionnaire results}

The scores obtained from the questionnaires after each condition are reported in Table 3. The analysis with Friedman ANOVAs reported that the arm/cylinder was embodied differently by the participants while experiencing the different conditions $\left(\chi^{2}=39.29 ; p<\right.$ $0.00001)$. In particular, the illusion of ownership of the avatar's arm in the $S$ condition was stronger than the sense of ownership of a cylinder $(p=0.000004)$ and also of the same virtual arm in the A condition $(p=0.00002)$. Irrespective of the synchronous or asynchronous movement, the virtual arm was more embodied than the cylinder, as the significant difference between A and CI shows $(p<0.0005)$. Also, the Friedman ANOVA conducted on the hand/cylinder embodiment showed significant differences for the reported level of ownership among conditions $\left(\chi^{2}=38.50 ; p<0.00001\right)$. In particular, the sense of ownership of the virtual hand was found to be significantly stronger in the $\mathrm{S}$ condition compared both to A $(p=0.000008)$ and CI conditions $(p=0.000003)$ and also higher in the A condition compared to the cylinder $(p=0.0074)$. Importantly, the synchronous movement of the finger not only led to the ownership of the hand but it extended to the entire arm.

A significant effect was found between Condition and the attention scores $\left(\chi^{2}=7.93 ; p=0.047\right)$, but not for anxiety scores $\left(\chi^{2}=6.30 ; p=0.097\right)$. However, post-hoc tests failed to report any significant difference between conditions in terms of attention paid to the painful stimulus (all $p^{\prime} \mathrm{s}>0.0083$ : CO vs. CI, $p=0.76$; $\mathrm{CO}$ vs. A, $p=0.029$; $\mathrm{CO}$ vs. S, $p=0.012$; CI vs. A, $p=0.11$; CI vs. S, $p=0.027$; A vs. S, $p=0.50)$. This would rule out a differential role of attention on the modulation of pain thresholds.

Table 3 Questionnaire scores (mean \pm standard error) reported per condition.

\begin{tabular}{lllll}
\hline Condition & Anxiety & Attention & Presence & Arm/Cylinder embodiment \\
\hline CO & $2.79 \pm 1.32$ & $3.88 \pm 1.68$ & - & - \\
Cl & $2.50 \pm 1.35$ & $3.71 \pm 1.76$ & $4.46 \pm 1.67$ & $1.83 \pm 1.40$ \\
A & $2.75 \pm 1.57$ & $3.00 \pm 1.44$ & $5.17 \pm 1.31$ & $3.54 \pm 1.61$ \\
S & $2.08 \pm 1.21$ & $2.83 \pm 1.58$ & $6.13 \pm 1.03$ & $6.21 \pm 0.83$ \\
\hline
\end{tabular}


The analysis of the 'presence' score (the illusion of being in the virtual space) highlighted a significant effect of Condition $\left(\chi^{2}=27.35 ; p<0.00001\right)$. Post-hoc tests revealed that the sense of presence reached in the $\mathrm{S}$ condition was significantly higher than both in $\mathrm{CI}$ $(p=0.00029)$ and in A $(p=0.00030)$; also, the presence in A was higher than in CI $(p=0.0161)$.

\section{Discussion}

The aim of the present study was to investigate whether the analgesic effects of seeing one's own limb are transferable also to a fake limb after a bodyownership illusion. Specifically, we explored heat pain threshold under different experimental conditions involving ownership of a virtual arm. We found a significant increase in the pain threshold with respect to the baseline conditions only in the case where the virtual and the real hand moved synchronously. Synchronous stimulation meant that the passive displacement of the participant's finger and the proprioceptive information that it evokes was congruent with the movement of the virtual finger that was visually perceived. Indeed, synchronous multisensory stimulation is known to induce an illusion of ownership of the virtual arm and thus of ownership of the virtual body part (Sanchez-Vives et al., 2010). No significant alteration of pain threshold occurred just by being immersed in the virtual environment. Furthermore, simply watching a virtual arm per se, i.e., the A condition, did not significantly affect the pain threshold compared with the baselines. Previous evidence outside VR has demonstrated that there is an analgesic effect of seeing a body, an effect that is restricted to the vision of one's own body (Longo et al., 2009). Here, we extend these findings by demonstrating that looking at a fake body may also be analgesic, as long as it is perceived as one's own. Since synchronous multisensory stimulation, along with first-person perspective (Slater et al., 2008; Sanchez-Vives et al., 2010), is a powerful inducer of ownership of a virtual body, that would be the virtual counterpart to seeing one's own body. Recently, it has been reported that the illusion of owning a rubber hand does not induce any significant change in the perception of pain (Mohan et al., 2012). Likewise, we found no difference in pain threshold between the $S$ and A conditions. However, contrary to the S condition, the pain threshold recorded during the A condition did not significantly differ from those of the other conditions (i.e., CO and CI). Importantly, our design allowed the disclosure of significant differences between the $S$ condition and the two main control conditions, namely the vision of virtual non-corporeal objects and the absence of VR.

It is well known that attention is an important modulator of pain perception (McCaul and Malott, 1984; Villemure and Bushnell, 2002; Johnson, 2005; Ossipov et al., 2010). VR experiences can be highly immersive and interactive and they work well to decrease pain sensation through a decreased attention to the own body (Hoffman et al., 2001; Malloy and Milling, 2010). Although our participants spontaneously reported that both asynchronous and synchronous conditions were the most distractive ones with respect to the pain stimulation, no significant differences in self-reported levels of attention paid to the painful stimulus were found among conditions (Table 3). As aforementioned, the asynchronous condition did not result in a significant modulation of pain thresholds compared with any other condition, in spite of the subjective reports of a decreased attention to the pain stimulation. These observations suggest that the difference in the pain threshold reported by the synchronous condition was probably not due to mere attentional processes. Nevertheless, it has to be acknowledged that self-reported measures of attention, although widely used in cognitive science, lack objectivity. The introduction of a concurrent cognitive task, e.g., a temporal order judgement (see, e.g., Spence and Parise, 2010), could eventually provide further and more objective insights to this extent. A caveat to the introduction of a parallel task though, is that this may interfere with the establishment of the illusion of ownership.

As well as for attention, our findings support that the feeling of 'presence' in VR, i.e., the illusory sensation of being in the virtual room and not in the laboratory, is not a major modulatory factor of the pain threshold. Participants reported a significantly higher sense of presence in $\mathrm{S}$ condition compared with either A and CI, and of A compared with CI. This is of interest because it evidences the relevance of owning a body in VR in order to increase the experience of being in the virtual world. The sense of presence in VR has been decomposed into the illusions of being in the virtual place and the plausibility of the situation (Slater, 2009). Having a body that is seen from a first-person perspective and with appropriate sensorimotor correlations seems to add to the plausibility. In our study, the differences in presence were not accompanied by differences in pain thresholds across conditions. Other authors have found a positive correlation between the feeling of presence and the decrease of pain threshold (Hoffman et al., 2004), given that higher presence can result in less attention to the actual body. However, in 
the study of Hoffman and colleagues, no virtual body was present; therefore, participants could not experience the virtual body ownership of an avatar's body in the virtual world. Moreover, their task was clearly distractive.

Anxiety has been notoriously linked to pain perception (Jones and Zachariae, 2002; Colloca and Benedetti, 2007), however we observed no significant differences in the level of anxiety among conditions. Furthermore, the adaptation to painful stimuli was cancelled out by balancing the order of the conditions across participants. Hence, we believe that, in the current experiment, other mechanisms intervened in the modulation of pain, in particular related to the ownership of artificial body parts.

To our knowledge, this is the first study with objective evidence on the relationship between virtual body ownership and pain threshold. In a previous work, we demonstrated that the variation of the colour of the embodied arm affects pain threshold. However, body ownership was not differentially manipulated, as all the experimental conditions implied the ownership of the avatar's limb (Martini et al., 2013). In a recent study, Hansel et al. (2011) investigated whether the pressure pain threshold varied during states of illusory dislocation of one's body, which implied an autoscopic phenomenon, i.e., seeing the fake body in a different location (out-of-body experience). To induce (or not) the illusion, tactile stimulation on the back of the participants was synchronously (or asynchronously) provided with respect to the touch that they saw either on a mannequin's back or on a non-corporeal object, visualized in front of the participant. The authors found that the pain threshold increased in the conditions where the mannequin was visualized but could not find a specific effect of the out-of-body illusion, i.e., identification with the mannequin, on the pain thresholds. Our results, instead, show that the heat pain threshold increases only when there is ownership of the avatar's limb. This said, due to important differences in the experimental paradigm, caution should be taken when comparing our results with the ones from Blanke's group (Hansel et al., 2011). Indeed, while these authors explored pain threshold on relation to self-location and self-identification with a dummy body placed in front of the subject, we explored the ownership of a collocated virtual body seen from a first-person perspective, a feature that has been shown to be a key factor in inducing virtual body ownership (Slater et al., 2010). Also, their smaller participants' sample may have not provided them enough statistical power. Another crucial difference may rely upon the distance between the body area where the multisensory stimulation was applied (i.e., on the participant's back) and the location where the pressure pain was induced (index finger). Actually, the strength of the ownership illusion decreases when moving away from the stimulation area (Tsakiris et al., 2006) and psychologically induced cooling of the body is strictly limited to the limb interested by the illusion (Moseley et al., 2008). These findings suggest that the effect on pain threshold may be spatially limited to an area proximal to the stimulated area.

Even if the rubber and virtual hand illusions take only a few seconds to work after stimulation onset (Ehrsson et al., 2005; Lloyd, 2007; Perez-Marcos et al., 2012), our results suggest that physiological changes may require longer duration under the illusion. This is in line with a previous rubber hand illusion study where changes in the limb skin temperature were observed after at least $5 \mathrm{~min}$ of stroking (Moseley et al., 2008). In our case, the shorter overall stimulation time may have prevented from any change on skin temperature. Therefore, inducing significant variations in skin temperature or pain threshold may need some tens of seconds to occur after the illusion starts to be experienced. It could also be that, the recording of the temperature of the left limb (control limb), compared with the temperature recorded in the right (experimental) limb, might have disclosed significant differences in the temperatures of the two limbs.

Although sensory mismatches reduce the strength of the virtual body ownership illusion (Perez-Marcos et al., 2012), the mere vision of a collocated virtual body seen from a first-person perspective is enough to induce the illusion to some extent (Slater et al., 2010). In the experiment described here, the subjective illusion of ownership was greater in the synchronous than in the asynchronous condition. However, the difference in the pain thresholds observed between the synchronous and asynchronous conditions did not reach the statistical significance. The strong perceptive cue that the first-person perspective provides could be the cause. Moreover, a few works on body ownership with healthy subjects have recently suggested that the ownership of a fake limb could involve the disownership of the corresponding real body part (e.g., Moseley et al., 2008; Guterstam et al., 2011). However, the actual occurrence of this disownership process has been lately questioned (de Vignemont, 2011). We think that the pain threshold effect found in the current study should be attributable to the ownership of the virtual limb and not to the disownership of the real limb based upon different facts. On the one hand, this would be in agreement with studies where the 
vision of one's own limb - no disownership is thus implied - is analgesic (Longo et al., 2009, 2012). On the other hand, recent findings show that somatosensory alterations following body ownership would not involve disownership (Folegatti et al., 2009; Llobera et al., 2013b). Moreover, we did not observe any change in either hand's or arm's skin temperature, while disownership has been associated to a temperature drop (Moseley et al., 2008).

At last, neither in the present experiment nor in the previous similar experiments (Longo et al., 2009, 2012; Hansel et al., 2011; Mohan et al., 2012), the role of emotional arousal has been taken into account. What is known is that if the owned body part is threatened, one tends to react as if the threat was directed to the real body (Slater et al., 2010; Kilteni et al., 2012). The investigation of affective response during the ownership of a new body could help in understanding the relationship between body and emotions not only within a single individual but also in a social context. For instance, given the proven ability that people have to share the emotional components of pain (Singer et al., 2004), future studies might investigate the affective reactions and the neurophysiological responses to an avatar in pain or under threat conditions, having previously manipulated the level of relationship (good or bad, strong or weak, etc.) of the real subject with the avatar.

\section{Conclusions}

In summary, we investigated the effects of the ownership of a digital body on thermal pain threshold. Our results show that the ownership of an avatar's limb can significantly increase the thermal pain threshold in that limb. Given the modulatory effect that the vision and the visual alteration of the affected real body part has on acute (Hoffman et al., 2011; Longo et al., 2009, 2012; Mancini et al., 2011) and chronic pain (Ramachandran et al., 2009; Ramachandran and Seckel, 2010), our findings may be relevant for the development and improvement of digital solutions for the treatment and rehabilitation from both acute and chronic pain states, by the application of customized visual feedback over the owned virtual body.

\section{Acknowledgements}

We are sincerely grateful to Ausias Pomes for the development of the finger tracking in VR and for his technical assistance. We also want to thank Dr. Jordi Serra for providing the thermal stimulation apparatus.

\section{References}

Bantick, S.J., Wise, R.G., Ploghaus, A., Clare, S., Smith, S.M., Tracey, I. (2002). Imaging how attention modulates pain in humans using functional MRI. Brain 125, 310-319.

Botvinick, M., Cohen, J. (1998). Rubber hands 'feel' touch that eyes see. Nature 391, 756.

Cole, J., Crowle, S., Austwick, G., Slater, D.H. (2009). Exploratory findings with virtual reality for phantom limb pain; from stump motion to agency and analgesia. Disabil Rehabil 31, 846-854.

Colloca, L., Benedetti, F. (2007). Nocebo hyperalgesia: How anxiety is turned into pain. Curr Opin Anaesthesiol 20, 435-439.

Ehrsson, H.H., Holmes, N.P., Passingham, R.E. (2005). Touching a rubber hand: Feeling of body ownership is associated with activity in multisensory brain areas. $J$ Neurosci 25, 10564-10573.

Folegatti, A., de Vignemont, F., Pavani, F., Rossetti, Y., Farnè, A. (2009). Losing one's hand: Visual-proprioceptive conflict affects touch perception. PLoS One 4, e6920.

Gillies, M., Spanlang, B. (2010). Comparing and evaluating real time character engines for virtual environments. Presence-Teleop Virt 19, 95-117.

Guterstam, A., Petkova, V.I., Ehrsson, H.H. (2011). The illusion of owning a third arm. PLOS ONE 6, el7208.

Hansel, A., Lenggenhager, B., von Kanel, R., Curatolo, M., Blanke, O. (2011). Seeing and identifying with a virtual body decreases pain perception. Eur J Pain 15, 874-879.

Hoffman, H.G., Doctor, J.N., Patterson, D.R., Carrougher, G.J., Furness, T.A. 3rd (2000a). Virtual reality as an adjunctive pain control during burn wound care in adolescent patients. Pain 85, 305-309.

Hoffman, H.G., Patterson, D.R., Carrougher, G.J. (2000b). Use of virtual reality for adjunctive treatment of adult burn pain during physical therapy: A controlled study. Clin J Pain 16, 244-250.

Hoffman, H.G., Patterson, D.R., Carrougher, G.J., Sharar, S.R. (2001). Effectiveness of virtual reality-based pain control with multiple treatments. Clin J Pain 17, 229-235.

Hoffman, H.G., Sharar, S.R., Coda, B., Everett, J.J., Ciol, M., Richards, T., Patterson, D.R. (2004). Manipulating presence influences the magnitude of virtual reality analgesia. Pain 111, 162-168.

Hoffman, H.G., Chambers, G.T., Meyer, W.J. 3rd, Arceneaux, L.L., Russell, W.J., Seibel, E.J., Richards, T.L., Sharar, S.R., Patterson, D.R. (2011) Virtual reality as an adjunctive non-pharmacologic analgesic for acute burn pain during medical procedures. Ann Behav Med 41, 183-191.

Johnson, M.H. (2005). How does distraction work in the management of pain? Curr Pain Headache Rep 9, 90-95.

Jones, A., Zachariae, R. (2002). Gender, anxiety, and experimental pain sensitivity: An overview. J Am Med Womens Assoc 57, 91-94.

Kalckert, A., Ehrsson, H.H. (2012). Moving a rubber hand that feels like your own: A dissociation of ownership and agency. Front Hum Neurosci 6,40 .

Kilteni, K., Normand, J.-M., Sanchez-Vives, M.V., Slater, M. (2012). Extending body space in immersive virtual reality: A very long arm illusion. PLOS ONE 7, e40867.

Llobera, J., González-Franco, M., Perez-Marcos, D., Valls-Solé, J., Slater, M., Sanchez-Vives, M.V. (2013a). Virtual reality for assessment of patients suffering chronic pain: A case study. Exp Brain Res 225, 105-1 17.

Llobera, J., Sanchez-Vives, M.V., Slater, M. (2013b). The relationship between virtual body ownership and temperature sensitivity. $J R$ Soc Interface 10, 20130300.

Lloyd, D.M. (2007). Spatial limits on referred touch to an alien limb may reflect boundaries of visuo-tactile peripersonal space surrounding the hand. Brain Cogn 64, 104-109.

Longo, M.R., Betti, V., Aglioti, S.M., Haggard, P. (2009). Visually induced analgesia: Seeing the body reduces pain. J Neurosci 29, 1212512130.

Longo, M.R., Iannetti, G.D., Mancini, F., Driver, J., Haggard, P. (2012). Linking pain and the body: Neural correlates of visually induced analgesia. J Neurosci 32, 2601-2607.

Malloy, K.M., Milling, L.S. (2010). The effectiveness of virtual reality distraction for pain reduction: A systematic review. Clin Psychol Rev 30, 1011-1018. 
Mancini, F., Longo, M.R., Kammers, M.P., Haggard, P. (2011). Visual distortion of body size modulates pain perception. Psychol Sci 22, 325330 .

Martini, M., Perez-Marcos, D., Sanchez-Vives, M.V. (2013). What color is my arm? Changes in skin color of an embodied virtual arm modulates pain threshold. Front Hum Neurosci 7, 438.

McCaul, K.D., Malott, J.M. (1984). Distraction and coping with pain. Psychol Bull 95, 516-533.

Mohan, R., Jensen, K.B., Petkova, V.I., Dey, A., Barnsley, N., Ingvar, M., McAuley, J.H., Moseley, G.L., Ehrsson, H.H. (2012). No pain relief with the rubber hand illusion. PLoS One 7(12), e52400.

Moseley, G.L., Olthof, N., Venema, A., Don, S., Wijers, M., Gallace, A., Spence, C. (2008). Psychologically induced cooling of a specific body part caused by the illusory ownership of an artificial counterpart. Proc Natl Acad Sci U S A 105, 13169-13173.

Murray, C.D., Pettifer, S., Howard, T., Patchick, E.L., Caillette, F., Kulkarni, J., Bamford, C. (2007). The treatment of phantom limb pain using immersive virtual reality: Three case studies. Disabil Rehabil 29, 1465-1469.

Ossipov, M.H., Dussor, G.O., Porreca, F. (2010). Central modulation of pain. J Clin Invest 120, 3779-3787.

Perez-Marcos, D., Sanchez-Vives, M.V., Slater, M. (2012). Is my hand connected to my body? The impact of body continuity and arm alignment on the virtual hand illusion. Cogn Neurodyn 6, 295-305.

Ramachandran, V.S., Brang, D., McGeoch, P.D. (2009). Size reduction using mirror visual feedback (MVF) reduces phantom pain. Neurocase 15, 357-360.

Ramachandran, V.S., Seckel, E.L. (2010). Using mirror visual feedback and virtual reality to treat fibromyalgia. Med Hypotheses 75 (6), 495-496.

Sanchez-Vives, M.V., Slater, M. (2005). From presence to consciousness through virtual reality. Nat Rev Neurosci 6, 332-339.

Sanchez-Vives, M.V., Spanlang, B., Frisoli, A., Bergamasco, M., Slater, M. (2010). Virtual hand illusion induced by visuomotor correlations. PLoS One 5, el0381.
Sato, K., Fukumori, S., Matsusaki, T., Maruo, T., Ishikawa, S., Nishie, H., Takata, K., Mizuhara, H., Mizobuchi, S., Nakatsuka, H., Matsumi, M., Gofuku, A., Yokoyama, M., Morita, K. (2010). Nonimmersive virtual reality mirror visual feedback therapy and its application for the treatment of complex regional pain syndrome: An open-label pilot study. Pain Med 11, 622-629.

Singer, T., Seymour, B., O’Doherty, J., Kaube, H., Dolan, R.J., Frith, C.D (2004). Empathy for pain involves the affective but not sensory components of pain. Science 303, 1157-1162.

Slater, M. (2009). Place illusion and plausibility can lead to realistic behaviour in immersive virtual environments. Philos Trans $R$ Soc Lond B Biol Sci 364, 3549-3557.

Slater, M., Perez-Marcos, D., Ehrsson, H.H., Sanchez-Vives, M.V. (2008). Towards a digital body: The virtual arm illusion. Front Hum Neurosci 2, $1-8$.

Slater, M., Spanlang, B., Sanchez-Vives, M.V., Blanke, O. (2010). First person experience of body transfer in virtual reality. PLoS One 5 , el0564.

Spence, C., Parise, C. (2010). Prior-entry: A review. Conscious Cogn 19 364-379.

Tecchia, F., Carrozzino, M., Bacinelli, S., Rossi, F., Vercelli, D., Marino, G., Gasparello, P., Bergamasco, M. (2010). A flexible framework for widespectrum VR development. Presence-Teleop Virt 19, 302-312.

Tsakiris, M., Prabhu, G., Haggard, P. (2006). Having a body versus moving your body: How agency structures body-ownership. Conscious $\operatorname{Cog} n 15$, 423-432.

de Vignemont, F. (2011). Embodiment, ownership and disownership. Conscious Cogn 20, 82-93.

Villemure, C., Bushnell, M.C. (2002). Cognitive modulation of pain: How do attention and emotion influence pain processing? Pain 95, 195199.

Yarnitsky, D., Sprecher, E., Zaslansky, R., Hemli, J.A. (1995). Heat pain thresholds: Normative data and repeatability. Pain 60, 329332 